\title{
Forthcoming smart DC nano-grid for green buildings-A reflective vision
}

\author{
M. H. Shwehdi, M. A. Al Aqil* \\ College of Engineering, King Faisal University, Hofuf-31982, Saudi Arabia
}

\begin{abstract}
Many small scale renewable generators generate low voltage DC power. AC main networks are supplied from costly and inefficient power convertors. Ultimately, energy produced from renewable resources is delivered to a DC load. The proposed solution in this paper concentrates on omitting the usage of costly and inefficient power converters in feeding DC networks, links, and devices from DC power supplies. The smart home consists of a home area network with a smart meter and intelligent devices. However, Wireless nodes are used to control and manage smart homes through intelligent and efficient algorithms. Such algorithm will be developed to control loads during peak hours, to interconnect smart meters and intelligent devices and power flow of loads. The total load of the building will be categorized and managed. Intelligent devices are connected to each load, monitoring and controlling the power flow individually. These intelligent devices can communicate with the smart meter, so that the load is controlled during peak hours based on the decision made by the algorithms.
\end{abstract}

Keywords: Zero net energy (ZNE), nano- grid, home network, home energy management, smart meter, intelligent devices, grid-wise architecture council's (GWAC)

\section{Introduction}

Increases in global energy costs, coupled with a need to reduce harmful fossil-based emissions, are calling for a worldwide clean and efficient energy sources and architectures. It is a fact that almost all residential and commercial buildings are fossil-fuels energy dependants; a concept that has been gaining popularity in recent years is the zero net energy (ZNE) buildings.

A ZNE building could significantly cut/reduce dependence on fossil-based energy and supply the required energy through on-site distributed generation, such as solar, wind, fuel cells, or micro-turbines. Recent regulatory from the state of California that require all new residential constructions be "Zero net energy" by 2020 — and all new commercial buildings by 2030 [1] have added further urgency to the drive for energy self-sufficient buildings.

Simply, the goal of achieving a ZNE building with a reasonable payback period is big challenge due to a numerous of active and passive technologies involved, including the selection of electrical technologies that consume less energy (high-efficiency appliances, HVAC, and lighting); efficient distribution architecture to cut power losses; portable energy storage for energy buffering; and the integration of renewable, such as solar, wind, and geothermal energy.

The key to realizing a cost-effective ZNE building is to reduce the consumption house's loads net energy. When the energy consumption is reduced, distributed generation and electrical wiring is needed, which directly results in reduced building costs and a shorter payback period for the landlords.

In this paper, a ZNE structure is proposed which includes enabling technology feed direct current (DC) to residential distribution. Through the use of high-efficiency electronics and bus architecture, a DC distribution system reduces the amount energy consumed, consequently, reducing the amount of the required renewable generation on-site, resulting in a cost-effective ZNE residence.

\footnotetext{
* Manuscript received July 9, 2014; revised September 3, 2014.

Corresponding author: M. H. Shwehdi; Tel.: + 0966 548087370; E-mail address: mshwehdi@kfu.edu.sa.

doi: $10.12720 /$ sgce.4.1.52-58
} 
Nowadays, conventional houses are fed from an alternating current (AC) electrical source. However, at the same time, many appliances and lighting technologies at these houses are natively DC loads, such as televisions, computers, LED lights, electric vehicles, batteries, fuel cells, and renewable sources. These and other many appliances are fed from a multistage power-conversion supply that first rectifies the incoming AC into DC. Usually, this is followed by a second DC-to-DC converter stage that converts the rectified DC voltage into a lower regulated voltage as required by the end load (e.g., 12VDC or 5VDC in personal computers). These conversion stages result in waste of electricity in the form of heat.

The efficiency of the majority of these power supplies usually varies between $70 \%$ and $75 \%$. The average efficiency of all power supplies, as estimated by Lawrence Berkeley National Lab (LBNL), is around 68\% [1]. Some high-end power supplies classified by the EPA as "80 Plus" may offer efficiencies greater than $80 \%$, although legacy systems offer much lower efficiency, especially at lower loads. However, in a conventional house, native AC motor-driven loads also exist. Similarly, the rectifier in the first stage is followed by a DC-to-AC inverter that drives the motor.

Renewable energy sources, such as solar and energy storage elements like batteries, are also essentially DC systems. Multistage power conversions are again needed to integrate them into the conventional AC distribution system. When integrating into an AC system, the DC output from a solar panel is first converted into another DC voltage, followed by an inverter that interfaces into the AC grid. The AC voltage produced by solar and battery inverters must be synchronized to the AC grid before they can be interconnected.

While many electric loads are native DC loads, then why not feeding them directly from a DC source in order to make them more efficient by reducing the number of power-conversion stages? This leads to reduction of losses from the conversion stage. Although a limited number of DC-to-DC or DC-to-AC conversions would remain, the input rectifier would be eliminated. Some of these DC-to-DC converters have peak efficiencies as high as $98 \%$. The resulting reduction in energy consumption directly translates to reduced costs and volume of renewable resources as well as energy storage required to supply the desired energy.

\section{Modern DC House Concept}

The application of DC house concept is new and gaining popularity. This concept depends on building a power distribution system around DC instead of the conventional AC system. This is because the DC house is a miniature grid in itself, comprised of DC loads and sources, as some entities call it a "DC nanogrid". The DC house can be fed from the AC electric utility grid (i.e., grid-parallel operating mode) or can be intentionally disconnected from the grid to function as a self-sustaining ZNE house.

There are many DC distribution configurations possible; one example is as shown in Fig. 1 below. A typical DC house could consist of two DC voltage buses, a 380VDC for high-power appliances, such as HVAC, washers, and dryers, and a lower 24VDC bus for smaller appliances and lighting. The 24VDC is stepped down from 380VDC and could be distributed throughout the house as a separate power bus (in addition to the 380VDC), or it could be in the form of dedicated power supplies that step down 380VDC to 24VDC for individual applications. A recent study by "Opportunities for Energy Savings Residential DC Power Bus" pointed out that in the short term, the latter option would offer a faster cost payback [2].

The DC house would contain different types of renewable energy sources, such as solar panels, which could be connected directly into the 380VDC bus, as would an energy-storage system, such as portable batteries. The energy storage device would serve as a back-up power supply to the house in case of any failure/trip in electric utility power supply and also support the loads when the DC house is intentionally operating in an island mode (self-sufficient, standalone zero-energy operation). Since the DC source is connected into a DC bus directly, there is no need for synchronization as required in AC systems. All the lighting would be DC-based LEDs as many of the commercial LED lamps in market operate from $24 \mathrm{~V}$ DC. In addition, the usage of electric vehicles in more common nowadays as they contain batteries which can be charged more efficiently from a DC source that already exists in the DC house. 


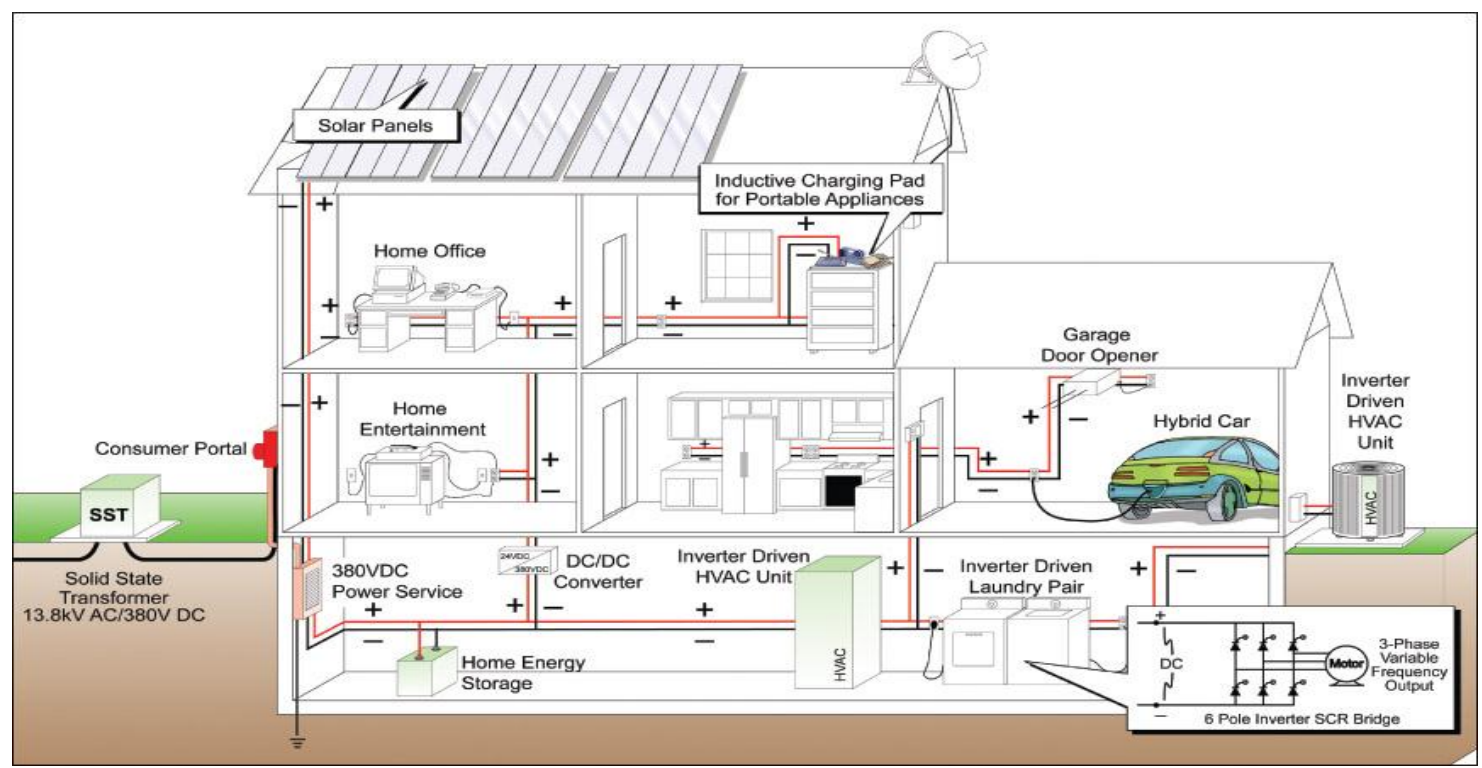

Fig. 1. Concept of DC house.

Today, the DC house is supplied by a conventional distribution transformer which step down the electric utility voltage of $13.8 \mathrm{kVAC}$ to $240 / 480 \mathrm{VAC}$. After that, a rectifier will convert the 240/480VAC to 380VDC. In the future, the DC house could be powered by smart and highly efficient solid-state transformers, currently under development for future development, which will convert $13.8 \mathrm{kVAC}$ directly to $380 \mathrm{VAC}$ to reduce the losses of rectifiers/inverters.

Since the DC house is designed to be fully supplied from local distributed generation sources when intentionally disconnected from the grid, it is essentially a ZNE building. Therefore, there is no need for modifications to reach the energy-natural state as in the conventional AC connections. The energy consumed by a DC house is less than that for a conventional AC house; due to high efficient appliances and power converters used in the DC house. This means that less energy is produced by the integrated distributed sources, and the distributed sources can be downsized relative to the requirements of an AC house. Low powered distributed resources translate into reduced capital costs. In fact, the application of DC houses cut off the payback period to a lower range and could considerably improve the possibility of widespread implementation of ZNE houses. In addition, to achieve ZNE architecture, passive design techniques could be used to supplement the system, as has been envisioned in standard AC-fed houses.

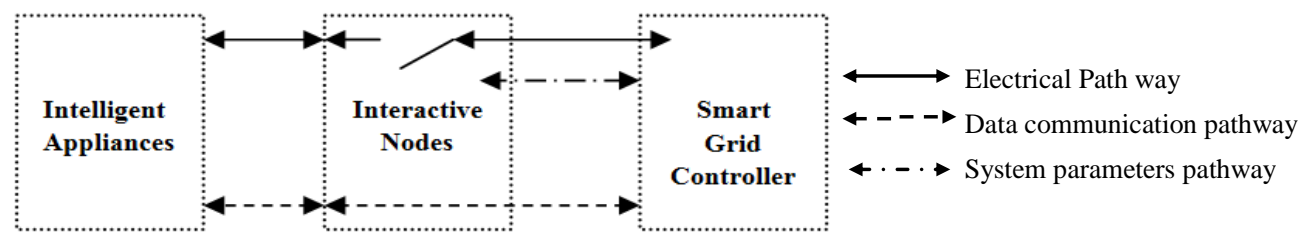

Fig. 2. Smart electrical system.

\section{Smart Grid Visualization \& Implementation}

The proposed system is as shown in Fig. 2. It consists of three main elements, the intelligent appliances, the interactive nodes and the computerized control mechanism where communication is present between these elements.

In the case of connecting an appliance to the node, the switch on the electrical pathway will remain in the open position. Then, the controller will detect that an appliance had been connected and it will send a signal through the communication channel requesting for identification data. If the controller assesses that 
the appliance can work properly at that node without adverse affects on any other nodes it sends back a signal to close the switch and allows the appliance to operate.

\subsection{The loads - Intelligent appliances}

Each DC appliance will need an electronic identifier which will contain its identifier parameters and have a communication channel to pass on this data to the system controller. Besides its basic voltage and current characteristics, there are many other parameters that are provided by each manufacturer for both safe operation and dynamic control. At this time the use of National Instruments (NI) specification hardware is a tested method to provide this type of intelligence to the appliances.

Normally, in an AC $230 \mathrm{~V}$ house the mode of operation is either on, power flows, or off, no power. However, in the DC house it is possible that at times of low power generation, circuit overload, or if it is running on emergency generation, partial power may be lost to the system. In such case, decisions have to be made as to which appliances should have priority to use the available power. Within a conventional home system, the homeowner would have to decide on which appliances to give priority and go around the house and physically unplug those that are of low priority. However, this issue is solved by applying the smart control system method where this can be done automatically using a hierarchical identifier. Such an identifier prioritises each appliance in order of criticality to the user which will be represented by a numbering system. For example, general category of emergency lighting may be assigned as number 1 , loads like internet/telephony as number 2, refrigerator/freezer as number 3, other lighting as number 4 and so on. Then, within each category there will be subcategories depending on importance. This hierarchical identifier should be part of an international agreed numbering system (ISO), perhaps in bands, but with the ability of additional control by the homeowner.

The smart meter collects the data of power consumption of the dishwasher, $\mathrm{TV}$, and the refrigerator, and also sends the control commands to them if necessary. The data generated by the smart meters in different buildings is transmitted to a data aggregator, which serves as a gateway/access point, routed to the electric utility or the distribution substation. Fig. 3 belows shows a typical application of such a smart meters [3].

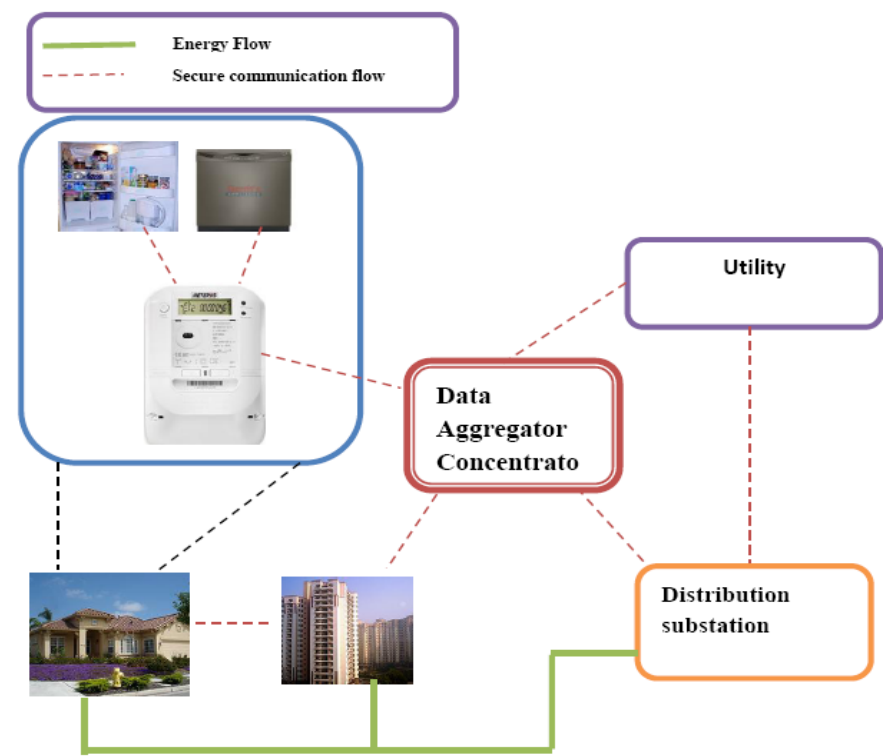

Fig. 3. An example of the smart metering structure.

\subsection{The nodes}

In most conventional houses operating on conventional AC power, there is a need of AC to DC conversion and a step-down transformer. These transformers have an associated carbon footprint, as well 
as using up energy in operation, which can be detected by the heat they give off. In the application of DC houses, the DC to DC converter function will be removed from the appliance and placed in the nodes instead. It has been shown that there are up to 25 gadgets on average (in 2002) per home [4]-[5]. Therefore by taking this function out of the appliance, a large amount of carbon footprint of the appliance will be reduced as well as a reduction in the amount of DC converters used in the house.

This will result in converting these nodes from being in passive mode as they are in a conventional AC system to being in active mode in the DC system. They incorporate DC to DC converters, measurement electronics as well as the three distinct pathways/channels mentioned above. The DC to DC converters must be isolated from the electrical mains so long as there is no current flowing to an appliance otherwise energy will leak away all the time. Real time measurement of the relevant system parameters is carried out by an embedded module in the node.

\subsection{The control mechanism}

The control mechanism has to receive on the demand side, data from the loads and from the electronic module in the nodes, and on the supply side from the voltage regulation system. It has to provide active and intelligent control of power flows to each node on each cable. A standard dashboard type interface much similar to that provided for a national smart grid control system will be needed.

In times of emergency when supply is reduced a priority powering down protocol must be enforced. This will see power to the least important nodes being cut first with a gradual withholding of energy to the less critical nodes, balancing the system so that the most critical nodes being given priority and only being powered down last, the protocol prioritizing according to the hierarchical identifier number of each load.

\subsection{Mechanism of an intelligent appliance operation}

- When a load is connected to a node, the mains electricity switch remains open while the communications channel is closed.

- A signal is sent to the appliance to read its parameters

- A decision is made based on measurements of all the other system parameters if it is safe to allow this appliance connectivity.

- If yes, the electrical pathway is opened and the device goes operational.

- If no, the electrical connectivity is denied by continuing to keep the electrical switch open. To operate the appliance another node must be provided.

- On removal of the load the electrical pathway switch should automatically open again.

ZigBee is a wireless technology which is designed for radio-frequency applications that require a low data rate, long battery life, and secure networking. It might be one of the most widely used communication technologies in the customer home network. The ZigBee and ZigBee smart energy profile (SEP) have been defined as the one of the communication standards for use in the customer premise network domain of the SG by the U.S. National Institute of Standards and Technology (NIST) [6]. It has also been selected by many electric utilities as the communication technology for the smart metering devices [7], since it provides a standardized platform for exchanging data between smart metering devices and appliances located on customer premises. The features supported by the SEP include demand response, advanced metering support, real time pricing, text messaging, and load control. This paper proposes the general problems of residential energy management and how power management equipment, in combination with smart appliances and home networks can address these problems. It describes one particular device and architecture as a comprehensive case study. A potential Smart Grid system architecture with consumer premises monitoring and control is shown in Fig. 4 [8]. It examines the problem of demand response on time scales of hours, seconds, milliseconds, and minutes and how this equipment can work both independently and in connection with a grid provider. This paper presents these elements within the context of the Grid-wise architecture council's (GWAC) stack framework focusing on basic connectivity and interoperability. 


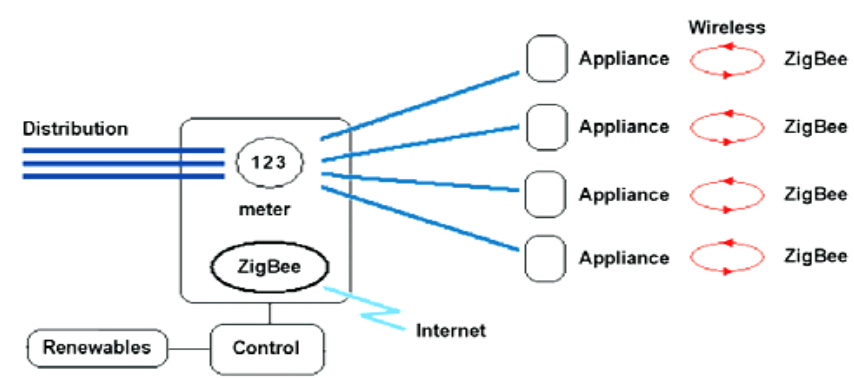

Fig. 4. Smart Grid system architecture with consumer Premises monitoring and control.

\subsection{Breaking new ground}

What about standards, codes, and personnel safety? An open industry association called the E-Merge Alliance is already addressing this and other issues, including ground-fault protection and arc-flash. The E-Merge Alliance is developing standards that could lead to the rapid adoption of DC power distribution in commercial buildings [6]. This group includes several industry vendors and services, electric utility representation through EPRI, and product safety testing and certification organizations like UL. The first standard to be released by E-Merge is "24VDC for Commercial Spaces." Used safely in the telecom industry for a long time, safe practices for 48VDC systems have been developed for these systems and can be adopted in DC houses. In DC data centres, the industry has agreed upon 380VDC as a standard voltage, organized as a split $\pm 190 \mathrm{VDC}$ system, to ensure safety. A similar configuration could be adopted in residences. In addition, OEMs are developing connectors that would allow safe disconnection and connection to DC receptacles.

In recent years there has been growing interest in the use of DC in the home, partly because many modern home appliances use DC voltage and most renewable energy sources generate DC power. By using DC voltage as the mains electricity system, the multiple stage energy conversions associated with a conventional AC system, which is fed from DC micro generators, are eliminated. This includes the expensive inverter which is always needed when DC micro generators feed into an AC electrical system. Therefore by exchanging the conventional AC-to-DC converters with integrated circuit DC-to-DC converters there should be the added benefit of a consequential saving in energy conversion losses, a reduction in the use of raw materials in their manufacture and therefore a reduction in the carbon footprint of the home. Different design implementations/scenarios have been investigated [5] from which it has been shown that an extra low voltage home of below 50V DC is possible. However there are constraints associated with voltage drops along the cables that reduce the operability of the home. To overcome these problems a smart grid as part of an integrated smart house in envisioned.

The DC house is not an alternative to a conventional AC house, and cannot at this time supersede it. However what the DC house can do, is help to bring a degree of energy independence with security, when implemented in the form of a hybrid AC/DC house and for the millions of households in the developing world that are not connected to an electric grid, and who may have to wait a long time until this may be possible, the DC house can provide a standalone solution now, which will increase their standard of living now. The quicker electricity is available to a society the faster GDP will grow [9]-[10].

\section{Conclusions}

A DC house is a new concept when distributes power system is built around DC instead of conventional AC system. The proposed Zero net energy (ZNE) building is enabling technology to feed direct current (DC) to residential distribution. Through the use of high-efficiency electronics, bus architecture each DC appliance will need an electronics identifier which will contain its identifier parameters and have a communication of channel to pass on this data to the system controller. Many companies have manufactured different types of hardware and protocols. Zero net energy (ZNE) building is being widely utilized for many reasons. Countries are constructed DC nano-network for smart residential and 
commercial buildings. Standards, codes and personnel safety have been achieved by E-merge alliance including. Such standards will lead to rapid adoption of DC power distribution in more buildings. Recent regulatory from California, USA that requires all new residential construction will be ZNE by 2020 and all new commercial buildings by 2030. Elimination of the high costs of inverters/converters when utilize AC to DC, as well as reduction of pollution caused by the ZNE building is to reduce the net energy consumed by the house loads. As energy consumption is reduced a smaller portion of the distributed generation and electrical wiring needed, which directly translates into reduction of building costs and a shorter payback period for the owner. ZNE building could significantly cut/reduce fossil based energy supply.

\section{Acknowledgment}

The authors appreciate the publication support provided by King Faisal University through the College of Engineering.

\section{References}

[1] Rajagopalan S., Fortenbery. B. Realizing a cost-effective zero net energy buildings through direct current (DC) residential distribution. EPRI, Jul. 2011.

[2] King D.J. Brodrick J.. Opportunities for energy savings? Residential DC power bus. Featured in the September 2010 edition of the ASHRAE Journal, 2010.

[3] X F., et al. Smart grid - the new and improved power grid: A survey. September 30, 2011.

[4] Kinn M.C . Proposed components for the design of a smart nano-grid for a domestic electrical system that operates at below 50v DC. 2nd IEEE-PES Manchester, UK; Dec 5-7, 2011.

[5] Kinn M.C. Benefits of direct current electricity supply for domestic applications. Master of Philosophy. School of Electrical and Electronics Engineering Faculty of Engineering and Physical Sciences. The University of Manchester; 2011:165.

[6] National Institute of Standards and Technology. (January 2010). NIST framework and roadmap for smart grid interoperability standards, release. [Online]. Available: http://www.nist.gov/publicaffairs/releases/upload/smartgridinteroperability final.pdf

[7] Farhangi H. The path of the smart grid. IEEE Power and Energy Magazine, 2010; 8(1):18-28.

[8] EMERGEALLIANCE. An open industry association. [Online]. Available: http://www.emergealliance.org/Home.aspx

[9] Yang H.Y. A note on the casual relationship between energy and GDP in Taiwan. Energy Economics, 2000; 22:309-317.

[10] Morimoto R,. Hope C. The impact of electricity supply on economic growth in Sri Lanka. Energy Economics, 2004; $26: 77-85$. 